068

\title{
MICOSI SUPERFICIALI E CUTANEE
}

\section{Migliozzi A., Cocco M.P.}

\section{Dipartimento di Patologia Clinica, P.O. S. Maria delle Grazie} ASL NA2

Materiali e metodi: i prelievi sono effettuati utilizzando, a seconda delle sede della lesione, un bisturi, una lancetta tagliente o una forbicina sterili. Il materiale prelevato è raccolto in una capsula di Petri sterile per essere esaminato al microscopio ottico dopo trattamento con $\mathrm{KOH}$.

L'esame colturale si esegue deponendo il materiale prelevato sui terreni di coltura rappresentati da Sabouraud classico o addizionato con cloramfenicolo e gentamicina per tutti i miceti e Sabouraud addizionato con cloramfenicolo e actidione selettivo per i Dermatofiti.

Per l'identificazione di alcuni Dermatofiti si utilizza il terreno all'Ureasi

Risultati: 68 campioni sono risultati positivi su un totale di 138 prelievi.

- Per un totale di 28 scraping cutanei, 7 sono risultati positivi, identificando:

3 T. rubrum, 3 A. niger, 1 M. canis

- Per un totale di 40 scraping ungueali delle mani e dei piedi, 29 sono risultati positivi, identificando:

5 C. albicans, 3 T. rubrum, 3 C. glabrata, 2 C. krusei, 2 C. parapsilosis, 2 C. tropicalis, 10 T. mentagrophytes, $1 \mathrm{~A}$. flavus, 1 Trichosporum spp.

\section{Conclusioni:}

La creazione di una raccolta di vetrini fissati e colorati per l'osservazione microscopica, di un album di immagini fotografiche si ritengono di estrema utilità quali parametri di riferimento per la consultazione di routine e l'approfondimento sistematico delle micosi superficiali e cutanee. 